\title{
S-Mono form Modules
}

\author{
Inaam, M.A.Hadi, Hassan K. Marhoon \\ Department of Mathematics, College of Education for Pure Science, \\ University of Baghdad (Ibn-Al-Haitham)
}

\begin{abstract}
In this paper, we introduce the concept S-monoform modules as a generalization of monoform modules. We study this class of modules, also we give several properties of these module and other related modules.

Key Words: Monoform module, small monoform module, S-monoform module, small submodule, small prime module, uniform module, S-uniform module, quasi-Dedekind module, hollow module.
\end{abstract}

\section{Introduction}

Let $\mathrm{R}$ be a commutative ring with unity and let $\mathrm{M}$ be a unitary $\mathrm{R}$-module, $\mathrm{M}$ is called a monoform module if for each nonzero submodule $\mathrm{N}$ of $\mathrm{M}$ and for each $\mathrm{f} \in \operatorname{Hom}(\mathrm{N}, \mathrm{M}), \mathrm{f} \neq 0$ implies $\operatorname{ker} f=(0)$, [15]. Equivalently $M$ is monoform if and only if every nonzero submodule $N$ of $M$ is rational, that is $N \leq K \leq M$, $\operatorname{Hom}\left(\frac{\mathrm{N}}{\mathrm{K}}, \mathrm{M}\right)=0,[14]$.

The concept small monoform appeared in [5] where an R-module $\mathrm{M}$ is called small monoform if for each $0 \neq N \leq M$ and for each nonzero $f \in \operatorname{Hom}(N, M)$, implies ker $\mathrm{f} \ll \mathrm{N}$. Also this class of modules studied in [8]. In this paper we introduce another generalization of monoform. $\mathrm{M}$ is called S-monoform module if for each nonzero small submodule $\mathrm{N}$ of $\mathrm{M}$ and for each nonzero $\mathrm{f} \in \operatorname{Hom}(\mathrm{N}, \mathrm{M})$, implies $\mathrm{ker} f=0$. And a proper submodule $\mathrm{N}$ of $\mathrm{M}$ is called small (denoted by $\mathrm{N} \ll \mathrm{M}$ ) if $\mathrm{N}+\mathrm{K} \neq \mathrm{M}$ for any proper submodule, [9].

We give the basic properties of S-monoform module and their relationships with small monoform, monoform module and other related modules.

\section{S-Mono form Modules-Basic Results}

In this section, as a generalization of monoform modules, S-monoform modules are introduced. Basic properties of S-monoform modules are given (see theorem (1.4)).

\section{Definition (1.1):}

Let $\mathrm{M}$ be an R-module. $\mathrm{M}$ is called $\mathbf{S}$-monoform if for each $\mathrm{N} \ll \mathrm{M}, \mathrm{N} \neq(0)$ and $\mathrm{f} \in \operatorname{Hom}(\mathrm{N}, \mathrm{M})$ implies ker $\mathrm{f}=(0)$.

A ring $\mathrm{R}$ is called $\mathbf{S}$ - monoform if it is $\mathrm{S}$-monoform $\mathrm{R}$-module.

\section{Remarks and Examples (1.2):}

(1) It is clear that $Z_{4}$ as $Z$-module is $S$-monoform.

(2) $\mathrm{Z}_{8}$ as $\mathrm{Z}$-module is not $\mathrm{S}$-monoform, since there exists $\mathrm{f}:<\overline{2}>\longrightarrow \mathrm{Z}_{8}$, such that $f(\overline{\mathrm{x}})=2 \overline{\mathrm{x}}$, for each $\overline{\mathrm{x}} \in<\overline{2}>$ and hence ker $\mathrm{f}=\{\overline{0}, \overline{4}\} \neq(\overline{0}) \neq(0)$. Also notice that $\mathrm{Z}_{8}$ is small monoform. Thus small monoform does not imply S-monoform.

(3) Clearly every monoform module is $\mathrm{S}$-monoform, but the converse is not true. For example: $\mathrm{Z}_{4}$ as $\mathrm{Z}$-module is S-monoform, but not monoform.

(4) If $\mathrm{M}$ is semisimple, then $\mathrm{M}$ is $\mathrm{S}$-monoform.

Proof:

As $M$ is semisimple, (0) is the only small submodule of $M$. Hence the result follows directly. In particular each of $Z_{6}, Z_{10}, Z_{2} \oplus Z_{2}$ as $\mathrm{Z}$-module is $\mathrm{S}$-monoform.

(5) The epimorphic image of S-monoform modules not necessarily S-monoform, for example: the Z-module $Z$ is S-monoform. But $\pi: \mathrm{Z} \longrightarrow \mathrm{Z} / 8 \mathrm{Z} \cong \mathrm{Z}_{8}$, where $\pi$ is the natural epimorphism. However $\mathrm{Z}_{8}$ as $\mathrm{Z}$-module is not $S$-monoform (see remarks and examples (1.2)(2)).

(6) Every nonzero submodule of S-monoform module is S-monoform module.

Proof: 
Let $\mathrm{M}$ be an S-monoform R-module and let $(0) \neq \mathrm{N} \leq \mathrm{M}$, for any $(0) \neq \mathrm{U} \ll \mathrm{N}$, let $\mathrm{f}: \mathrm{U} \longrightarrow \mathrm{N}, \mathrm{f} \neq(0)$. Consider the diagram

$$
\mathrm{U} \stackrel{f}{\longrightarrow} \mathrm{N} \stackrel{\mathrm{i}}{\longrightarrow} \mathrm{M}, \mathrm{i} \circ \mathrm{f} \neq(0)
$$

Where $\mathrm{i}$ is the inclusion mapping $\mathrm{N}$ to $\mathrm{M}$. But $\mathrm{U} \ll \mathrm{N}$, implies $\mathrm{U} \ll \mathrm{M}$. Hence ker (i॰f) $)(0$ ), since $\mathrm{M}$ is $\mathrm{S}$ monoform. But ker $\mathrm{f} \subseteq$ ker (iof), so ker $\mathrm{f}=(0)$. Thus $\mathrm{N}$ is $\mathrm{S}$-monoform.

Note (1.3):

The direct sum of S-monoform modules is not necessarily S-monoform module. Now, consider the following example:

Let $\mathrm{M}=\mathrm{Z}_{4} \oplus \mathrm{Z}_{4}$ as $\mathrm{Z}$-module, let $\mathrm{N}=\langle\overline{2}>\oplus<\overline{2}>\square \mathrm{M}$ and let $\mathrm{f}: \mathrm{N} \longrightarrow \mathrm{M}$ defined by $f(\overline{\mathrm{x}}, \overline{\mathrm{y}})=(\overline{\mathrm{x}}, 2 \overline{\mathrm{y}})$, for each $(\overline{\mathrm{x}}, \overline{\mathrm{y}}) \in \mathrm{N}$, ker $\mathrm{f}=\{(\overline{0}, \overline{0}),(\overline{0}, \overline{2})\} \neq\{(\overline{0}, \overline{0})\}$, then $\mathrm{M}$ is not $\mathrm{S}$-monoform, but $\mathrm{Z}_{4}$ as $\mathrm{Z}$-module is $\mathrm{S}$ monoform (see remarks and examples (1.2) (1)).

Recall that an R-module $\mathrm{M}$ is called fully stable if for each $\mathrm{N} \leq \mathrm{M}, \mathrm{N}$ is stable; that is for each $\mathrm{f} \in \operatorname{Hom}(\mathrm{N}, \mathrm{M}), \mathrm{f}(\mathrm{N}) \subseteq \mathrm{N}$, see [1]. Equivalently $\mathrm{M}$ is fully stable if and only if $\underset{\mathrm{M}}{\operatorname{ann}}(\underset{R}{\operatorname{ann}}(\mathrm{x}))=(\mathrm{x})$, for each $\mathrm{x} \in$ M, see [1,corollary $3.5, \mathrm{p} .22]$.

\section{Theorem (1.4):}

Let $\mathrm{M}=\mathrm{M}_{1} \oplus \mathrm{M}_{2}, \mathrm{M}_{1}, \mathrm{M}_{2} \leq \mathrm{M}$ such that for each $\mathrm{f}: \mathrm{N}_{1} \oplus \mathrm{N}_{2} \longrightarrow \mathrm{M}, \quad \mathrm{f} \neq 0$ implies $\mathrm{f}\left(\mathrm{N}_{1}\right) \neq(0)$, $f\left(N_{2}\right) \neq 0$ (i.e. $f\left|N_{1} \neq 0, f\right| N_{2} \neq 0$ and $M$ is fully stable, then $M_{1}$ and $M_{2}$ are S-monoform if and only if $M$ is Smonoform.

\section{Proof:}

$(\Leftarrow)$ It is clear by remarks and examples $(1.2)(6)$.

$\left(\Rightarrow\right.$ Let $(0) \neq N \ll M$ and let $\mathrm{f} \in \operatorname{Hom}(\mathrm{N}, \mathrm{M}), \mathrm{f} \neq 0$. Since $\mathrm{M}$ is fully stable, $\mathrm{N}=\mathrm{N}_{1} \oplus \mathrm{N}_{2}$, where $\mathrm{N}_{1}=\mathrm{N} \cap \mathrm{M}_{1}$, $\mathrm{N}_{2}=\mathrm{N} \cap \mathrm{M}_{2}$. Moreover, $\mathrm{f}(\mathrm{N}) \subseteq \mathrm{N}$. As $\mathrm{N} \ll \mathrm{M}$, we get $\mathrm{N}_{1} \ll \mathrm{M}_{1}$ and $\mathrm{N}_{2} \ll \mathrm{M}_{2}$ by [2, proposition 5.20]. Let $\mathrm{g}_{1}=\mathrm{f} / \mathrm{N}_{1}: \mathrm{N}_{1} \longrightarrow \mathrm{M}, \mathrm{g}_{2}=\mathrm{f} / \mathrm{N}_{2}: \mathrm{N}_{2} \longrightarrow \mathrm{M}$. Again, since $\mathrm{M}$ is fully stable $\mathrm{g}\left(\mathrm{N}_{1}\right) \subseteq \mathrm{N}_{1}, \mathrm{~g}\left(\mathrm{~N}_{2}\right) \subseteq \mathrm{N}_{2}$. Thus $\mathrm{g}_{1}: \mathrm{N}_{1} \longrightarrow \mathrm{M}_{1}, \mathrm{~g}_{2}: \mathrm{N}_{2} \longrightarrow \mathrm{M}_{2}$ and so ker $\mathrm{g}_{1} \oplus$ ker $\mathrm{g}_{2}=\langle 0\rangle \oplus\langle 0\rangle=\langle 0>$. Now, let $\mathrm{n} \in \operatorname{ker} \mathrm{f} \leq \mathrm{N}$, then $\mathrm{n}=\mathrm{n}_{1}+\mathrm{n}_{2}$ for some $\mathrm{n}_{1} \in \mathrm{N}_{1}, \mathrm{n}_{2} \in \mathrm{N}_{2}$ and $\mathrm{f}(\mathrm{n})=0$. Thus $0=\mathrm{f}(\mathrm{n})=\mathrm{f}\left(\mathrm{n}_{1}\right)+\mathrm{f}\left(\mathrm{n}_{2}\right)=\mathrm{g}\left(\mathrm{n}_{1}\right)+\mathrm{g}\left(\mathrm{n}_{2}\right)$. Hence $\mathrm{g}\left(\mathrm{n}_{1}\right)=-\mathrm{g}\left(\mathrm{n}_{2}\right) \in \mathrm{N}_{1} \cap \mathrm{N}_{2}=(0)$, it follows that $\mathrm{g}\left(\mathrm{n}_{1}\right)=\mathrm{g}\left(\mathrm{n}_{2}\right)=0$; that is $\mathrm{n}_{1} \in \operatorname{ker} \mathrm{g}_{1}=(0), \mathrm{n}_{2} \in \operatorname{ker} \mathrm{g}_{2}=(0)$. Therefore $\mathrm{n}_{1}+\mathrm{n}_{2}=0$ and hence $\operatorname{ker} \mathrm{f}=(0)$.

\section{Note (1.5):}

The condition $\mathrm{M}$ is fully stable in theorem (1.4) cannot be dropped, since the module $\mathrm{M}$ (in note (1.3)) is not fully stable, since for $\mathrm{W}=<\overline{2}>\oplus<\overline{0}>$, there exists $\mathrm{f}: \mathrm{W} \longrightarrow \mathrm{M}$ defined by $f(\overline{\mathrm{x}}, \overline{0})=(\overline{0}, \overline{\mathrm{x}})$, for each $(\overline{\mathrm{x}}, \overline{0}) \in \mathrm{W}$, then $\mathrm{f}(\mathrm{W})=<\overline{0}>+<\overline{2}>\not \mathrm{W}$.

\section{S-Monoform Modules and S-Uniform Modules}

It is known that monoform (small monoform) module implies uniform (see [3,theorem (2.3)] where an $\mathrm{R}$-module $\mathrm{M}$ is called uniform if every nonzero submodule $\mathrm{N}$ of $\mathrm{M}$ is essential (large), and a submodule $\mathrm{N}$ of $\mathrm{M}$, $\mathrm{N} \neq(0)$ is called essential (denoted by $\mathrm{N} \leq \mathrm{e}$ ) if $\mathrm{N} \cap \mathrm{W} \neq(0)$ for each $\mathrm{W} \neq(0)$, see [6]. However this is not true for S-monoform (see remarks and examples (1.2)(4)). However we introduce the concept of S-uniform and we see that there are some connections between S-monoform module and S-uniform module (see theorems $(2.5,2.13)$, propositions $(2.2,2.14)$ and corollary $(2.7)$.

\section{Definition (2.1):} M.

Let $\mathrm{M}$ be an R-module. $\mathrm{M}$ is called $\mathbf{S}$-uniform if every nonzero small submodule of $\mathrm{M}$ is essential in shows:

It is clear that every uniform module is S-uniform, but the converse is not true as the following example $\mathrm{Z}_{6}$ as $\mathrm{Z}$-module is $\mathrm{S}$-uniform, since $\mathrm{Z}_{6}$ has no nonzero small submodule. However $\mathrm{Z}_{6}$ is not uniform. 
Let $\mathrm{M}$ be an R-module, put $\mathrm{Z}(\mathrm{M})=\{\mathrm{m} \in \mathrm{M}: \underset{\mathrm{R}}{\operatorname{ann}}(\mathrm{M}) \underset{\mathrm{e}}{\leq \mathrm{R}}\}, \mathrm{Z}(\mathrm{M})$ is called a singular submodule of $\mathrm{M}$. $\mathrm{M}$ is called singular if $Z(M)=M$ and $M$ is called nonsingular if $Z(M)=(0)$, see [6].

\section{Proposition (2.2):}

\section{Proof:}

Let $\mathrm{M}$ be a nonsingular $\mathrm{R}$-module, if $\mathrm{M}$ is $\mathrm{S}$-uniform. Then $\mathrm{M}$ is $\mathrm{S}$-monoform.

Let $(0) \neq N \ll M$ and let $f \in \operatorname{Hom}(N, M), f \neq(0)$. To prove ker $f=(0)$. By $1^{\text {st }}$ Fundamental theorem $\mathrm{N} /$ ker $\mathrm{f} \cong \mathrm{f}(\mathrm{N})$. But $\mathrm{f}(\mathrm{N}) \subseteq \mathrm{M}$ and $\mathrm{M}$ is nonsingular, hence $\mathrm{f}(\mathrm{N})$ is nonsingular by [6,proposition 1.22,p.32]. Thus $\mathrm{N} /$ ker $\mathrm{f}$ is nonsingular. But ker $\mathrm{f} \subseteq \mathrm{N}$ and $\mathrm{N} \ll \mathrm{M}$, so ker $\mathrm{f} \ll \mathrm{M}$. As $\mathrm{M}$ is S-uniform we have ker $\mathrm{f} \leq \mathrm{M}$. Hence ker $\mathrm{f} \leq \mathrm{e}$. Also, since $\mathrm{N} \leq \mathrm{M}$, so $\mathrm{N}$ is nonsingular, hence $\mathrm{N} / \mathrm{ker} \mathrm{f}$ is singular by [6,proposition 1.21,p.32]. Thus $\mathrm{N} /$ ker $\mathrm{f}$ singular and nonsingular. It follows that $\mathrm{N} /$ ker $\mathrm{f}=(0)$; that is $\mathrm{N}=$ ker $\mathrm{f}$ and so that $\mathrm{f}=0$ which is a contradiction. Thus ker $\mathrm{f}=(0)$.

\section{Remark (2.3):}

The converse of proposition (2.2), is not true in general. For example:

Consider $Z_{12}$ as $Z$-module; $\left\langle\overline{6}>\right.$ is the only nonzero small submodule of $Z_{12}$, let $f:<\overline{6}>\longrightarrow Z_{12}, f \neq(0)$, then $\mathrm{f}$ is the inclusion mapping. Thus ker $\mathrm{f}=(0)$. Hence $\mathrm{Z}_{12}$ is $\mathrm{S}$-monoform. However $\mathrm{Z}_{12}$ is not $\mathrm{S}$-uniform, since $<\overline{6}>\ll Z_{12}$. But $<\overline{6}>\underset{\mathrm{e}}{\not} \mathrm{Z}_{12}$, since $<\overline{6}>\cap<\overline{4}>=(0)$.

\section{Corollary (2.4):}

\section{Proof:}

Let $\mathrm{M}$ be a nonsingular $\mathrm{R}$-module, if $\mathrm{M}$ is small monoform. Then $\mathrm{M}$ is $\mathrm{S}$-monoform.

Since $M$ is small monofrm, then $M$ is uniform by [8,proposition 1.6]. Hence $M$ is S-uniform and so by proposition (2.2), $\mathrm{M}$ is $\mathrm{S}-$ monoform.

Recall that $\mathrm{M}$ is an R-module, then $\mathrm{M}$ is monoform if and only if $\mathrm{M}$ is uniform prime, see [13,theorem 2.3]. We prove the following:

\section{Theorem (2.5):}

\section{Proof:}

If $\mathrm{M}$ is S-uniform and semiprime R-module, then $\mathrm{M}$ is $\mathrm{S}$-monoform.

Let $(0) \neq N \ll M$ and $f \in \operatorname{Hom}(N, M)$ such that $f \neq 0$. To prove ker $f=(0)$. Suppose ker $f \neq(0)$. As ker $\mathrm{f} \subseteq \mathrm{N} \ll \mathrm{M}$, ker $\mathrm{f} \ll \mathrm{M}$ and since $\mathrm{M}$ is S-uniform, then ker $\mathrm{f} \leq \mathrm{M}$. Since $\mathrm{f} \neq 0$, then there exists $\mathrm{x} \in \mathrm{N}$ such that $f(x) \neq 0$. Hence (ker $f) \cap<x>\neq(0)$; that is there exists $r \in R, r \neq 0$ such that $0 \neq r \quad x \in$ ker $f$. Thus $\mathrm{f}(\mathrm{rx})=\mathrm{rf}(\mathrm{x})=0$

On the other hand $\mathrm{N} \ll \mathrm{M}$, rx $\in \mathrm{M}$ implies $<\mathrm{r} \mathrm{x}>\ll \mathrm{M}$. But $\mathrm{M}$ is S-uniform, so that $<\mathrm{rx}>\leq \mathrm{e}$ and hence $<\mathrm{rx}>\cap<\mathrm{f}(\mathrm{x})>\neq(0)$. Then there exists $\mathrm{r}_{1} \in \mathrm{R}, \mathrm{r}_{1} \neq 0$ such that $\mathrm{r}_{1} \mathrm{rx} \neq 0$ and $\mathrm{r}_{1} \mathrm{rx} \in<\mathrm{f}(\mathrm{x})>$. This implies $r_{1} r x=c f(x)$, for some $0 \neq c \in R$. It is follows that $r_{1} r^{2} x=\operatorname{crf}(x)=0$. Thus $\left(r_{1} r\right)^{2} x=0$. Hence $r_{1} r x=0$, since $M$ is semiprime, which is a contradiction. Thus ker $\mathrm{f}=(0)$ and hence $\mathrm{M}$ is S-monoform.

\section{Remark (2.6):}

The converse of theorem (2.5), is not true. For example:

The $\mathrm{Z}$-module $\mathrm{Z}_{4}$ is S-monoform. But $\mathrm{Z}_{4}$ is not semiprime since $2^{2} \cdot \overline{1}=\overline{0}$, but $2 \cdot \overline{1} \neq \overline{0}$.

\section{Corollary (2.7):}

\section{Proof:}

If $\mathrm{M}$ is an $\mathrm{S}$-uniform and prime $\mathrm{R}$-module, then $\mathrm{M}$ is $\mathrm{S}$-monoform.

Since every prime module is semiprime, the result follows directly.

Recall that an R-module $M$ is called small prime if $\underset{R}{\operatorname{ann}} \mathrm{M}=\underset{R}{\operatorname{ann}} \mathrm{N}$ for each $\mathrm{N} \ll \mathrm{M}$, see [10].

To prove the next two corollaries, we need the folowng lemma: 


\section{Lemma (2.8):}

Let $M$ be a small prime. Then for each $x \neq 0$ with $(x) \ll M$ and for each $f \in \operatorname{Hom}((x), M)$ with $f \neq 0$, then ker $f=(0)$.

Proof:

Let $x \neq 0$ and let $(x) \ll M$, let $0 \neq f \in \operatorname{Hom}((x), M)$ and let $r x \in$ ker $f$, then $f(r x)=0$. This implies $\mathrm{r} f(\mathrm{x})=0$. But $\mathrm{M}$ is small prime; that is $(0)$ is a small prime submodule. Hence either $\mathrm{f}(\mathrm{x})=0$ or $r \in((0) \underset{R}{:} M)=\operatorname{annM}$. As $f(x) \neq 0$, we get $r \in \operatorname{annM}$. Thus $r x=0$, which implies ker $f=(0)$.

\section{Corollary (2.9):}

Let $\mathrm{M}$ be an R-module such that every submodule of $\mathrm{M}$ is cyclic and small. If $\mathrm{M}$ is small prime, then $\mathrm{M}$ is S-monform.

Proof:

It is follows by lemma (2.8).

Recall that an $\mathrm{R}$-module $\mathrm{M}$ is a hollow module if $\mathrm{M} \neq(0)$ and every proper submodule of $\mathrm{M}$ is small in $\mathrm{M}$, see [4].

\section{Corollary (2.10):}

Let $\mathrm{M}$ be a small prime such that every submodule is hollow and Noetherian R-module. Then M is Smonoform.

\section{Proof:}

Let $\mathrm{N} \leq \mathrm{M}$ and $\mathrm{N} \neq(0)$. Since $\mathrm{M}$ is Noetherian, then $\mathrm{N}$ is a finitely generated submodule of $\mathrm{M}$. But $\mathrm{M}$ is hollow, so that $N$ is cyclic submodule. Hence $N=(x)$, for some $x \in M, x \neq(0)$. Thus the result is obtained by lemma (2.8).

An R-module $\mathrm{M}$ is called quasi-Dedekind if every nonzero R-submodule $\mathrm{N}$ of $\mathrm{M}$ is quasi-invertable; that is $\operatorname{Hom}(\mathrm{M} / \mathrm{N}, \mathrm{M})=0$. A ring $\mathrm{R}$ is quasi-Dedekind if it is quasi-Dedekind R-module see [11,definition 1.1,p.24]. Equivalently $M$ is quasi-Dedekind module if and only if for each nonzero $f \in \operatorname{End}(M)$, $f$ is monomorphism see [11, theorem $1.5, \mathrm{p} .26]$.

The following proposition shows that S-monoform implies monoform under the class hollow quasiDedekind module.

\section{Proposition (2.11):}

Proof:

Let $\mathrm{M}$ be a hollow module and quasi-Dedekind R-module. If $\mathrm{M}$ is S-monoform, then $\mathrm{M}$ is monoform.

Let $(0) \neq N \leq M$ and let $f \in \operatorname{Hom}(N, M)$ with $f \neq 0$. If $N \neq M$. Since $M$ is hollow, then $N \ll M$. But $M$ is S-monoform by hypothesis, implies ker $f=(0)$. If $M=N$, then $\operatorname{ker} f=(0)$, since $M$ is quasi-Dedekind. Thus $M$ is monoform.

\section{Note (2.12):}

The condition $\mathrm{M}$ is quasi-Dedekind in proposition (2.11) is necessarily. For example: $\mathrm{Z}_{4}$ as $\mathrm{Z}$-module is S-monoform and hollow. Also it is not quasi-Dedekind and it is not monoform.

Under the class of fully stable modules, we have the following result:

\section{Theorem (2.13):}

\section{Proof:}

Let $\mathrm{M}$ be a fully stable R-module. If $\mathrm{M}$ is a small prime and S-uniform, then $\mathrm{M}$ is S-monoform.

Let $(0) \neq N \ll M$ and let $f \in \operatorname{Hom}(N, M)$ with $f \neq 0$. To prove ker $f=(0)$, suppose ker $f \neq(0)$. Since ker $\mathrm{f} \leq \mathrm{N} \ll \mathrm{M}$, then ker $\mathrm{f} \ll \mathrm{M}$. But $\mathrm{M}$ is S-uniform, so ker $\mathrm{f} \underset{\mathrm{e}}{\leq} \mathrm{M}$. Hence $<\mathrm{x}>\cap \operatorname{ker} \mathrm{f} \neq(0)$, for any $\mathrm{x} \in \mathrm{N}$, $x \neq 0$. This means there exists $r \neq 0,0 \neq r x \in$ ker $f$ which implies $0=f(r x)=r f(x)$. But $f \in \operatorname{Hom}(N, M)$ and $N$ is stable, so $\mathrm{f}(\mathrm{N}) \subseteq \mathrm{N}$, hence $<\mathrm{f}(\mathrm{x})>\subseteq \mathrm{N}$. But $\mathrm{N} \ll \mathrm{M}$, so $<\mathrm{f}(\mathrm{x})>\ll \mathrm{M}$. As $\mathrm{M}$ is small prime and $\mathrm{r} f(\mathrm{x})=0$, we get that either $f(x)=0$ or $r \in \operatorname{annM}$. But $x \notin$ ker $f$. Thus $r \in \operatorname{annM}$, so $r x=0$ which is a contradiction. Therefore ker $\mathrm{f}=(0)$.

Recall that a submodule $\mathrm{N}$ of an $\mathrm{R}$-module $\mathrm{M}$ is called rational in $\mathrm{M}$ if $\underset{\mathrm{R}}{\operatorname{Hom}}(\mathrm{X} / \mathrm{N}, \mathrm{M})=0$ for any $\mathrm{N} \leq \mathrm{X} \leq \mathrm{M}$, see [3]. 
It is known that every rational submodule is essential [3]. Also it is known that: $\mathrm{M}$ is monoform if and only if $\operatorname{Hom}(\mathrm{X} / \mathrm{N}, \mathrm{M})=0$ for each $\mathrm{N} \leq \mathrm{M}$ and for each $\mathrm{N} \leq \mathrm{X} \leq \mathrm{M}$, see [14].

We have the following result:

\section{Proposition (2.14):}

Let $\mathrm{M}$ be an R-module. If for each $\mathrm{N} \ll \mathrm{M}$, $\operatorname{Hom}(X / N, M)=0, N \leq X \leq M$ (i.e. for each $N \ll M, N$ is rational, then $\mathrm{M}$ is $\mathrm{S}$-monoform and S-uniform.

\section{Proof:}

Let $(0) \neq W \ll M$ and let $f \in \operatorname{Hom}(W, M)$ with $f \neq 0$. If $\operatorname{ker} f=(0)$, then nothing to prove. If ker $f \neq(0)$, then $\mathrm{W} /$ ker $\mathrm{f} \cong \mathrm{f}(\mathrm{W}) \subseteq \mathrm{M}$. Hence there exists an isomorphism g such that $\mathrm{g}: \mathrm{W} / \mathrm{ker} \mathrm{f} \longrightarrow \mathrm{f}(\mathrm{W})$. Consider the diagram

$$
\mathrm{W} / \operatorname{ker} f \stackrel{\mathrm{g}}{\longrightarrow} f(\mathrm{~W}) \stackrel{\mathrm{i}}{\longrightarrow} \mathrm{M}
$$

Where $\mathrm{i}$ is the inclusion mapping. Thus $\mathrm{i} \circ \mathrm{g} \in \operatorname{Hom}(\mathrm{W} / \mathrm{ker} \mathrm{f}, \mathrm{M})$ and $\mathrm{i} \circ \mathrm{g} \neq 0$. On the other hand ker $\mathrm{f} \leq$ $\mathrm{W} \ll \mathrm{M}$, so ker $\mathrm{f} \ll \mathrm{M}$. But $\operatorname{Hom}(\mathrm{W} / \mathrm{ker} \mathrm{f}, \mathrm{M}) \neq(0)$, so we get a contradiction with the hypothesis. Thus ker $\mathrm{f}=$ (0). Also for each $\mathrm{N} \ll \mathrm{M}$, then $\mathrm{N} \leq \mathrm{M}$, since $\mathrm{N}$ is rational submodule. Therefore $\mathrm{M}$ is $\mathrm{S}$-uniform.

Recall that an R-module $\mathrm{M}$ is called multiplication if for each submodule $\mathrm{N}$ of $\mathrm{M}, \mathrm{N}=\mathrm{IM}$ for some ideal I of R. Equivalently $M$ is multiplication R-module if for each submodule $N$ of $M, N=(N: M) M$, where $(\mathrm{N}: \mathrm{M})=\{\mathrm{r} \in \mathrm{R}: \mathrm{rM} \subseteq \mathrm{N}\}$, see [12].

Under the class of multiplication module we have the following result:

\section{Proposition (2.15):}

Let $\mathrm{M}$ be a multiplication R-module with $\underset{\mathrm{R}}{\operatorname{ann}}(\mathrm{M})$ is a prime ideal of $\mathrm{R}$. Consider the following:

(1) For each $\mathrm{N} \ll \mathrm{M}$, $\mathrm{N}$ is rational submodule.

(2) For each $\mathrm{N} \ll \mathrm{M}$, $\mathrm{N}$ is essential (i.e. $\mathrm{M}$ is S-uniform).

(3) $\mathrm{M}$ is $\mathrm{S}-$ monoform.

Then $(1) \Leftrightarrow(2)$ and $(1) \Rightarrow(3)$

Proof:

$(1) \Rightarrow(2)$ It is clear.

(2) $\Rightarrow(1)$ Let $(0) \neq N \ll M$. Suppose there exists $N \leq X \leq M$ such that $\operatorname{Hom}(X / N, M) \neq(0)$, then there exists $\mathrm{f} \in \operatorname{Hom}(X / N, M), f \neq 0$. So there exists $x+N \in X / N, x \notin N$ such that $f(x+N)=m \neq 0$. Since $N \underset{e}{M}$, there exists $\quad r \neq 0$ such that $0 \neq r x \in N$. It follows that $r m=r f(x+N)=f(r x+N)=0$, then $r m=0$. Since $M$ is multiplication $\mathrm{Rm}=<\mathrm{m}>=\mathrm{IM}$, for some ideal $\mathrm{I} \in \mathrm{R}$. Hence $\mathrm{rIM}=(0)$. Thus $\mathrm{rI} \subseteq \underset{\mathrm{R}}{\operatorname{ann}} \mathrm{M}$. But $\underset{\mathrm{R}}{\operatorname{ann}} \mathrm{M}$ is prime ideal, so either $r \in \underset{R}{\operatorname{ann}} M$ or $I \subseteq \underset{R}{\operatorname{ann}} M$. If $r \in \underset{R}{\operatorname{ann}} M$, then $r M=(0)$ which implies $r x=0$, which is a contradiction. Thus $I \subseteq \underset{R}{\text { ann }} M$, hence $<\mathrm{m}>=\mathrm{IM}=(0)$, which is a contradiction. Thus $\operatorname{Hom}(\mathrm{X} / \mathrm{N}, \mathrm{M})=(0)$, for each $\mathrm{N} \leq \mathrm{X} \leq \mathrm{M}$ (i.e. $\mathrm{N}$ is rational submodule).

(1) $\Rightarrow$ (3) It follows by proposition (2.14).

all

Recall that an R-module $M$ is called comonoform module if for every $N<M$, $\operatorname{Hom}(M, N / L)=(0)$, for

\section{Proposition (2.16):}

$\mathrm{M}$ is comonoform and S-monoform quasi-Dedekind R-module, then M is monoform.

\section{Proof:}

Since $\mathrm{M}$ is comonoform, then $\mathrm{M}$ is hollow by [7, lemma 17]. Byproposition (2.11), $\mathrm{M}$ is monoform. Now we introduce the following: 


\section{Definition (2.17):}

An-R-module $M$ is called small polyform if for each $N \ll M, N \neq(0), f \in \operatorname{Hom}(N, M), \operatorname{ker} f \underset{\mathrm{e}}{\not} \mathrm{N}$.

The following result explains some connection between S-monoform module and small polyform module.

\section{Proposition (2.18):}

Proof:

If $\mathrm{M}$ is S-monoform, then $\mathrm{M}$ is small polyform.

Let $(0) \neq N \ll M$ and $f \in \operatorname{Hom}(N, M), f \neq 0$. Since $M$ is S-monoform, ker $f=(0) \nsucceq N$. Thus $M$ is small polyform.

\section{Proposition (2.19):} Proof:

If $\mathrm{M}$ is small polyform and S-uniform, then $\mathrm{M}$ is S-monoform.

Let $(0) \neq N \ll M$ and $f \in \operatorname{Hom}(N, M)$ with $f \neq 0$. To prove ker $f=(0)$. Suppose $k e r f \neq(0)$. It is clear that ker $\mathrm{f} \leq \mathrm{N} \ll \mathrm{M}$, hence ker $\mathrm{f} \ll \mathrm{M}$. On the other hand, $\mathrm{M}$ is S-uniform implies ker $\mathrm{f} \leq \mathrm{M}$. But this contradits the hypothesis, $\mathrm{M}$ is small polyform. Thus ker $\mathrm{f}=(0)$ and so that $\mathrm{M}$ is $\mathrm{S}$-monoform.

Corollary (2.20):

If $\mathrm{M}$ is S-uniform, then $\mathrm{M}$ is small polyform if and only if $\mathrm{M}$ is S-monoform.

\section{References}

[1]. M.S.Abbas, "On Fully Stable Modules", Ph.D. Thesis, University of Baghdad, (1990).

[2]. E.W.Anderson and K.R.Fuller, "Rings and Categories of Modules", Springer-Verlage, New York, (1992).

[3]. N.V.Dung, D.V.Huynh, P.F.Smith and R.Wisbauer, "Extending Modules", John Wily and Sons, Inc. New York, (1994).

[4]. P.Fleury, "Hollow Modules and Local Endomorphism Rings", Pac.J. Math., Vol. 53, No.2 (1974), p.379-385

[5]. T.Y.Ghaw, "Some Generalizations of Quasi-Dedekind Modules", M.Sc. Thesis, University of Baghdad, Iraq, (2010).

[6]. K.R.Goodearl, "Ring Theory, Nonsingular Rings and Modules ", Marcel Dekker, Inc. New York and Basel, (1976).

[7]. G.Güngöroglu, "Copolyform Modules", Commun. Fac.Sci. Univ.Ank.Series A1, V. 49 (2000), pp.101-110

[8]. I.M.Ali Hadi and H.K. Marhoon, Small Monoform Modules, Ibn Al-Haitham Journal for Pure and Applied Sci. 4(1980), 2014.

[9]. F.Kasch, "Modules and Rings", Academic Press, Inc. London, (1982).

[10]. L.S.Mahmood, Small Prime Modules and Small Prime Submodule", Journal of Al-Nahrain University Science, Vol.15, No.4 (2012), pp.191-199.

[11]. A.S.Mijbass, "Quasi-Dedekind Modules", Ph.D. Thesis, University of Baghdad, Iraq, (1997).

[12]. P.F.Smith, "Some Remarks on Multiplication Modules", Arch.Math., 50, (1988), pp.223-235.

[13]. P.F.Smith, "Compressible and Related Modules, in Abelian Groups, Rings, Modules and Homological Algebra, eds", P.Goeters and O.M.G. Jend (Chapman and Hull, Boca Raton), (2006), pp.1-29.

[14]. H.H.Storrer, "On Goldman's Primary Decomposition", Lecture Notes in Math., Vol.246, Springer-Verlag, Berlin, Heidelberg, New York, (1972)

[15]. Zelmanowitze, J.M., "Representation of Rings with Faithful Polyform Modules", Comm. in Algebra, 14(6) (1986), p.11411169. 